\title{
Study protocol: The SunBurst trial-a register-based, randomized controlled trial on thoracolumbar burst fractures
}

\author{
Simon BLIXT ${ }^{1,2}$, Sebastian MUKKA ${ }^{3}$, Peter FÖRSTH ${ }^{4}$, Olof WESTIN ${ }^{5}$, \\ and Paul GERDHEM ${ }^{1,2}$ on behalf of the SunBurst study group ${ }^{a}$
}

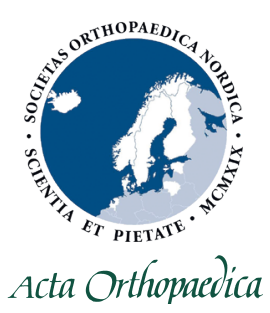

\begin{abstract}
${ }^{1}$ Department of Clinical Science Intervention and Technology, Karolinska Institutet, K54, Stockholm; ${ }^{2}$ Department of Reconstructive Orthopaedics, Karolinska University Hospital, Huddinge, Stockholm; ${ }^{3}$ Department of Surgical and Perioperative Sciences (Orthopaedics), Umeå University, Umeå; ${ }^{4}$ Department of Surgical Sciences, Uppsala University Hospital, Uppsala; ${ }^{5}$ Department of Orthopaedics, Institute of Clinical Sciences at Sahlgrenska Academy, University of Gothenburg, and Sahlgrenska University Hospital, Gothenburg, Sweden

a The SunBurst study group: Riyad Abd-Ali and Håkan Löfgren, Neuro-Orthopedic Center, Region Jönköping County, Jönköping, Sweden; Lukas Bobinski, Department of Surgical and Perioperative Sciences (Orthopaedics), Umeå University, Umeå, Sweden; Anastasios Charalampidis, Department of Reconstructive Orthopaedics, Karolinska University Hospital, Huddinge, Sweden; Ted Eneqvist, Department of Clinical Science and Education, Södersjukhuset, Stockholm, Sweden; Ralph Hasserius and Anders K Möller, Institution of Clinical Sciences (IKVM), Lund University and Department of Orthopedics, Skåne University Hospital, Malmö, Sweden; Mari Hänni and Monica Sjöholm, Department of Surgical Sciences, Uppsala University Hospital, Uppsala, Sweden; Anders Joelson and Freyr Gauti Sigmundsson, Department of Orthopedic Surgery, Örebro University Hospital, Örebro, Sweden; Martin Kempny, Department of Orthopedics, Halmstad County Hospital, Halmstad, Sweden; Per Näsman, KTH Royal Institute of Technology, Department of Real Estate and Construction Management, Centre for Safety Research, Stockholm, Sweden; Gylfi Ólafsson, Westfjords Healthcare Institute, Ísafjörður, Iceland; Anders Olai, Department of Spinal Surgery, Faculty of Health Sciences, Linköping University, Linköping, Sweden; Erik Vestberg, Department of Orthopedics, Kalmar County Hospital, Kalmar, Sweden.

Correspondence: simon.blixt@ki.se

Submitted 2021-10-04. Accepted 2021-12-29.
\end{abstract}

Background and purpose - The treatment for patients with thoracolumbar burst fractures without neurological deficit or complete rupture of the posterior ligament complex (PLC) is controversial and includes both surgical and non-surgical options. Current evidence on which treatment is optimal remains inconclusive. In this study we compare surgical with non-surgical treatment.

Methods - The study is a nationwide, multicenter, register-based randomized controlled trial (R-RCT). Patients with a thoracolumbar burst fracture will be identified by the Swedish Fracture Register. The admitting physician will be notified during the registration process and the patient will be screened for eligibility. Patients, 18 to 66 years old without neurologic deficit to more than a single nerve root and without complete rupture of the PLC, are eligible for the study. 202 patients will be randomized in a 1:1 relation to either surgical or non-surgical treatment. Patient-reported outcome measures (PROMs), including the Oswestry Disability Index (ODI) and radiological data, will be collected at the time of injury, after 3-4 months, and after 1 year. Additional data from national health registries will be collected after 1 year.

Outcome - The primary outcome is the ODI 1 year after injury. Secondary outcomes include additional PROMs, adverse events, drug consumption, sick leave, healthcare consumption, and imaging data.
Interpretation - The primary outcome is the ODI 1 year after injury. Secondary outcomes include additional PROMs, adverse events, drug consumption, sick leave, healthcare consumption, and imaging data.

Estimated duration - The study started on September 1, 2021 and will continue for approximately 4 years.

Trial registration - The trial is registered at www.clinicaltrials.com, NCT05003180.

Burst fractures, characterized by compression of both the anterior and middle column of the spine, most often occur in the thoracolumbar junction $(1,2)$. They can occur in all ages and are associated with pain, disability, and sick leave (3-9). Treatment options depend on several factors and include both surgical and non-surgical care $(\mathbf{1 0 , 1 1 )}$. In cases with no neurological deficit and without complete rupture of the posterior ligament complex (PLC), treatment options remain controversial (10-16). Most studies on thoracolumbar burst fractures have been observational and retrospective (16). The randomized controlled trials (RCTs) that have been carried out comparing surgical with nonsurgical treatment have been limited by small sample sizes and conflicting results $(\mathbf{1 7 , 1 8 )}$. To date, there is no consensus on which treatment is optimal for these patients $(\mathbf{1 0 , 1 4 , 1 9 - 2 2 )}$. 


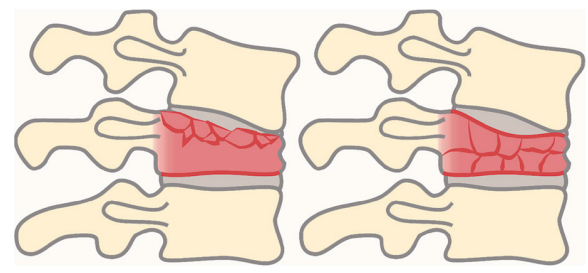

Figure 1. Drawings of burst fractures, defined as type $A 3$ (left) and A4 (right) according to the AOSpine thoracolumbar spine injury classification system. A3 involves a single endplate and A4 involves both endplates. Both the anterior and posterior wall of the vertebral body must be fractured to classify as a burst fracture. Drawing by Pontus Andersson.

Sweden has a long history of nationwide healthcare quality registers with more than 100 active registers today (23). The Swedish Fracture Register (SFR) started in 2011 and has since become a nationwide register for all types of orthopedic fractures (24). In recent years RCTs have been carried out with the aid of existing national quality register platforms. Today there are 2 ongoing register-based randomized controlled trials (R-RCTs) in cooperation with the SFR $(25,26)$. The StUdy oN Burst Fractures (SunBurst) will be the 3rd $\mathrm{R}-\mathrm{RCT}$ in cooperation with the SFR and the 1st R-RCT on vertebral fractures.

\section{Aim}

The purpose of the SunBurst trial is to compare surgical versus non-surgical treatment in patients who have sustained a thoracolumbar burst fracture without severe neurological deficit and without complete rupture of the PLC.

\section{Methods}

\section{Study design}

SunBurst is a nationwide, multicenter, open label, parallel assignment, R-RCT using the SFR as its study platform (27). Study protocol items where reported according to SPIRIT guidelines (28).

\section{Study subjects and eligibility criteria}

Inclusion criteria

- Patients between 18 and 66 years.

- A single level thoracolumbar burst fracture between T10 and L3 of type A3 or A4 according to the AO Spine classification proposed by Vaccaro et al. and Reinhold et al. in 2013 (Figure 1) (29,30).

- Individuals with minor fractures ${ }^{\mathbf{a}}$ in adjacent vertebrae are eligible for study inclusion if these fractures would not have resulted in any treatment.

- Diagnosis of burst fracture, no later than 2 weeks after injury.

- Informed consent for study participation.

\footnotetext{
a These include fractures of the transverse process, spinous process, lamina, facet, discrete vertebral body compression fractures, or vertebral body edema.
}

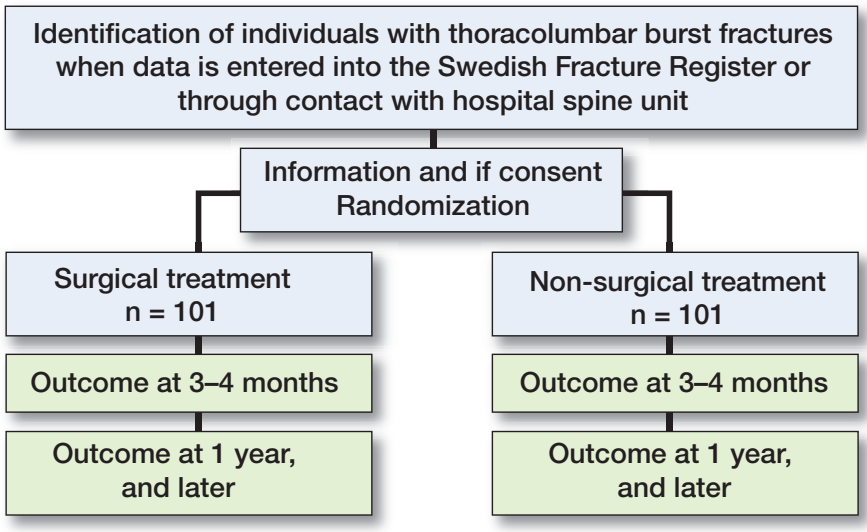

Figure 2. Study design register-based randomized controlled trial.

\section{Exclusion criteria}

- Unable to consent, no consent given or not informed.

- Neurological injury involving more than a single level nerve root, i.e., spinal cord and/or cauda equina injury.

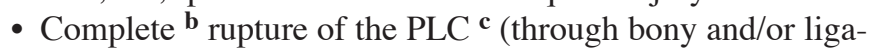
mentous structures) verified on MRI (31).

- Patients with ankylosing spinal disorders spanning the fracture area.

- Open vertebral fracture.

- Additional injury that would impair early ambulation, e.g., long bone fractures, severe head injury, long-lasting intensive care, etc.

- Individuals not deemed suitable for operative or nonoperative treatment due to comorbidities .

- Patients already included in the study cannot be randomized again if an additional spine fracture occurs.

The flow chart of the study is shown in Figure 2.

The trial will use the SFR study platform. SFR has nationwide coverage. Data in SFR is regularly entered by the treating physician. If data on a patient fulfills the inclusion criteria for SunBurst, the treating physician is notified on the SFR platform that this patient may fulfill the inclusion criteria. The treating physician is asked to notify the closest department that treats spine fractures. All hospital departments in Sweden that treat burst fractures surgically are represented in the SunBurst study group.

Study eligibility will be crosschecked by the spine consultant on call before screening and randomization is made. Randomization will be done within the SFR platform in a 1:1 manner after consent has been obtained. The randomization sequence is made in blocks without stratification by Uppsala Clinical Research center (https://www.ucr.uu.se) for a total of 232 individuals. Study inclusion will end when 101 individuals in both arms have been randomized. Since the study will compare surgical to non-surgical treatment blinding cannot be performed.

In the event a patient is not registered in the SFR by the treating physician at a hospital without a spine surgery service, it is

\footnotetext{
$\mathbf{b}$ The structures of the posterior ligamentous complex should be clearly visibly disrupted on the MRI scan to be classified as complete. Incomplete disruptions are eligible for the study.

c The posterior ligamentous complex consists of supraspinous ligaments, the interspinous ligaments, ligamentum flavum, facet capsules, and thoracolumbar fascia.
} 
our experience that a spine consultant on call will most likely be contacted to discuss treatment. The consultant will thereby be able to identify eligible patients and hopefully the losses of potential study patients will be minimized.

\section{Study interventions}

Surgical stabilization will be compared with nonsurgical treatment. The study is pragmatic, meaning that surgical technique, choice of implants, and details surrounding inpatient care are at the discretion of the treating physician.

\section{Surgical treatment}

Surgical stabilization is performed with posterior fixation with either an open or minimally invasive technique. The suggested technique is short segment fixation with pedicle screws inserted 1 level above and below the fractured vertebra, without fusion or decompression. If feasible, pedicle screws are inserted in the fractured vertebra. Fixation is established by connecting the pedicle screws to rods $(32,33)$. Surgery is to be performed within 2 weeks from the injury. A brace will not be used postoperatively. Early ambulation after surgery is encouraged. Physiotherapy and other measures of rehabilitation are prescribed on an individual basis. Surgery for removal of implants is optional and can be done according to local routines.

\section{Non-surgical treatment}

Early ambulation after randomization is encouraged. A standard 3-point (Jewett) brace or similar may be offered for up to 3 months for pain relief. The choice of supplier and brand of brace is decided by the treating physician. The brace will only be used upon mobilization. Brace usage will be estimated by the patient at the 3-4 months follow-up visit. Physiotherapy and other rehabilitation measures will be considered and prescribed on an individual basis.

\section{Treatment crossover control group}

In the uncommon event of neurological compromise after inclusion in the study, the reason for this will be assessed (34). If neurological compromise is due to canal compromise or progressing kyphosis, surgery is advisable. Inability to mobilize a patient due to intense pain despite adequate analgesics and possibly a brace may be a reason to conduct surgical treatment. A change in treatment will be documented in the SFR.

An increasing kyphosis without neurological deficit at follow-ups is not a reason for treatment change as no evident relation between kyphosis and patient-reported outcome has been described (34). Initial extent of canal compromise or an increase in canal compromise during follow-up is not a reason for treatment change in the absence of neurological symptoms (34).

\section{Study activities}

Clinical and radiological assessment, as well as patientreported outcome measures (PROMs), will be collected at the time of fracture, at 3-4 months, and at 1 year (Table 1). Longterm collection of PROMs may be performed 5 and 10 years after the fracture (Table 1).

\section{Withdrawal of patients from the trial}

Participants are free to withdraw from the trial at any time. Data already collected through the SFR will be retained in the study database. No additional data will be added, including data collected through crossmatching subjects with other registers. Withdrawn participants will not be replaced.

\section{Endpoints}

\section{Primary endpoint}

The primary outcome is to compare the score on the Oswestry Disability Index (ODI) between the surgically and non-surgically treated patients 1 year after the injury. ODI is a questionnaire that measures disability due to back pain. It has been frequently used since its publication in 1980 internationally and in Sweden (35-38). We will use the Swedish translation of version $2.1(36,39)$. The ODI consists of 10 items with 6 answers for each item. Each answer corresponds to a number between 0 and 5 , where 0 is no disability and 5 is maximum disability. The items are added and multiplied by 2 to generate an index from 0 to 100 . The level of disability ranges from no disability to bedbound, 0-20 no or minimal disability, 21-40 moderate disability, 41-60 severe disability, 61-80 crippled, 81-100 bedbound (40).

We assume that individuals before the injury have an ODI on a par with population-based data. At best, ODI after thoracolumbar fracture treatment approaches pre-fracture levels. 
Population-based data indicates a mean ODI of 8 for adults $(41,42)$. Information on ODI levels after thoracolumbar fractures are scarce. Mean ODI was 36 at 2 years after surgical treatment of thoracolumbar fractures in a study by Wei et al. (43). In a study by Möller et al. (7) of 27 non-surgically treated individuals mean ODI was 11 at 2 decades or more after a burst fracture. In the RCT by Wood et al. (8), ODI was 20 in the surgical group and 2 in the non-surgical group a mean 18 years after the burst fracture.

\section{Secondary endpoints}

Secondary objectives are to evaluate whether surgical compared with non-surgical treatment results in better healthrelated quality of life, shorter time to return to work, lesser consumption of analgesics, and fewer adverse events and costs associated with the treatment.

In addition to ODI, the patients will report other well-established PROMs, namely the Short Musculoskeletal Function Assessment (SMFA), which is an instrument measuring the functional status in patients with musculoskeletal disorders and EQ-5D-5L, including EQ-VAS, which is a standardized quality of life instrument developed by the EuroQol group (44-46).

Radiological data will be collected according to Table 1. All patients will undergo computed tomography (CT) of the thoracolumbar spine at the time of the injury, at 3-4 months, and at 1 year, magnetic resonance imaging (MRI) of the thoracolumbar spine at the time of injury and at 1 year, and a whole-spine standing weightbearing radiograph at 1 year. The degree of fracture comminution according to the load-sharing classification (47), degree of fracture compression, local and global kyphosis, adjacent segment degeneration, and extent of any soft tissue injuries will be registered.

The study participants will be crossmatched with other registers and databases by using their Swedish personal identity number (PIN). The National Board of Health and Welfare National Patient Register (NPR) will be used to compare the level of inpatient and outpatient healthcare, spinal surgery during follow-up, and adverse events such as postoperative infections and thromboembolism (48). Data on analgesics and antibiotics prescribed will be collected through the Swedish Prescribed Drug Register (PDR) (49). Mortality, including the cause of death, will be collected from the National Board of Health and Welfare Cause of Death Register (50).

In this study, adverse events will be defined as any undesirable event until the follow-ups $(\mathbf{2 0 , 5 1 )}$. The severity of the adverse events will be graded according to Landriel Ibañez et al. (52). Adverse events will be collected through the patient files, the SFR, the NPR (Appendix 1, see Supplementary data), and the PDR. Adverse events will be registered as surgical and medical complications. These will be analyzed as dichotomous variables with chi-square or Fisher's exact tests.

Data on any prescribed analgesics and antibiotics will be collected. Anatomical Therapeutic Chemical classification system (ATC) codes will be used to group the pharmaceuti- cals. Collected prescriptions will be assessed as dichotomous data (collected/not collected) at different time intervals: 0 to 4 months and 4 months to 1 year. To compare opioid prescriptions, conversion to morphine equivalents will be done.

Data from the Swedish Social Insurance Agency will be used to collect information regarding sick leave (53). Data will be stratified based on the presence or absence of sick leave before the fracture event. Data on total time on sick leave as well as the diagnosis used for sick leave will be collected.

Data on costs on the individual level will be collected and analyzed. This consists of costs for the inpatient and outpatient visits (including surgery, radiographs, braces), cost of pharmaceutical treatments, and cost for sick leave. The number of outpatient and telephone contacts (physician, nurse, physiotherapist) the fracture and treatment have resulted in will be registered from patient files and registries. Earnings lost in relation to the fracture will be estimated from the 'Longitudinal integrated database for health insurance and labour market studies' available at Statistics Sweden (54).

Quality-adjusted life years (QALYs) will be calculated for each group (as measured by EQ-5D-5L). Combining the cost of each treatment with the QALY yields the incremental costeffectiveness ratio (ICER), which we will use to compare the cost with other medical treatments

\section{Data collection}

Baseline data including screening questions, fracture classification, age, sex, type of trauma, time of diagnosis, and treatment intervention will be collected through the SFR and transcribed to the study database. ODI is not part of the SFR and will be collected by the central study coordinator. Written informed consent will be kept at each participating hospital and a copy at Karolinska University Hospital. Radiographs and hospital files will be collected from each study site and reviewed for surgical details. Study subjects will be crosschecked with other registers to collect additional data. Data collection is summarized in Table 2. The steering committee will supervise the study to verify that informed consent and all questionnaires are collected. The steering committee will have regular contacts with participating centers to confirm that they adhere to study protocol and will be available for discussion of inclusion and exclusion criteria. All original data will be stored on Karolinska University hospital computers and only available for the study's researchers, maintaining confidentiality in accordance with national data legislation. The authors will not have access to the complete dataset until all patients have been collected and no interim analysis will be made.

\section{Estimated sample size and power}

How large a difference in ODI should be between groups to be clinically relevant is not known. Often the ODI minimal clinically important difference (MCID) is used as an estimate of a clinically relevant group difference. In a study by Copay et al. (55), MCID for ODI was estimated to be around 10. In previ- 
Table 2. Data collection

\begin{tabular}{|c|c|c|c|c|c|}
\hline \multirow[b]{2}{*}{ Type of data } & \multicolumn{5}{|c|}{ Data collected by } \\
\hline & $\begin{array}{l}\text { Central } \\
\text { study } \\
\text { coordinator }\end{array}$ & $\begin{array}{l}\text { Swedish } \\
\text { Fracture } \\
\text { Register }\end{array}$ & $\begin{array}{c}\text { National } \\
\text { Patient } \\
\text { Register }\end{array}$ & $\begin{array}{c}\text { Prescribed } \\
\text { Drug } \\
\text { Register }\end{array}$ & $\begin{array}{l}\text { Agency/ } \\
\text { Statistics } \\
\text { Sweden }\end{array}$ \\
\hline ODI & $x$ & & & & \\
\hline SMFA & & $x^{a}$ & & EQ-5D-5L & $x^{a}$ \\
\hline Images & $\mathrm{x}$ & & & & \\
\hline Fracture classification & & $x$ & & & \\
\hline Screening question answer & & $\mathrm{X}$ & & & \\
\hline Additional spine surgery & & $\mathrm{X}$ & $\mathrm{x}$ & & \\
\hline Adverse events & & $\mathrm{X}$ & $\mathrm{X}$ & & \\
\hline Analgesics/antibiotics & & & & $\mathrm{X}$ & \\
\hline Sick leave data & & & & & $x$ \\
\hline Earnings & & & & & $x$ \\
\hline
\end{tabular}

a small difference between groups in ODI that is less than the estimation of MCID we will not be able to conclude that either treatment is superior to the other.

For secondary outcomes, similarly to the primary outcome, we will use Student's t-test to test differences in continuous variables measured on at least interval scale between the groups. Visual estimation will be used to assess normality of the data. If the data does not approximate a normal distribution the Mann-Whitney test will be used. In order to evaluate hypotheses of variables in contingency tables, the chi-square test will be used or, in the case of small, expected frequencies, Fisher's exact test. Multivariate logistic regression analysis will be used for exploratory purposes with PROMs as the dependent variable. ODI will be dichotomized as no disability (0-20) and disability (21-100). The other PROMs will

ous studies the standard deviation (SD) for ODI is between 10 and 20, with lower values seen after surgical treatment of thoracolumbar fractures than after degenerative lumbar spine conditions $(43,55)$.

To identify a minimal clinically important difference (MCID) in the ODI of 10 with a standard deviation of 20 with the probability of a type 1 error (alpha) set to 0.05 and power of $90 \%$, a sample size of 84 patients in each group is needed. To account for a loss to follow-up of up to $20 \%, 101$ patients in each group are needed in the RCT.

\section{Statistics}

Data will be presented as means with $95 \%$ confidence intervals for data with normal distribution, medians and interquartile ranges for data that is not normally distributed, and numbers or proportions for categorical data.

Data from the different groups will be compared based on the "intention to treat" principle (ITT). All patients, regardless of treatment change, loss to follow-up or dropout, remain in the analysis of the group to which they were randomized. Sensitivity analyses will be performed comparing the ITT data against the per-protocol data, i.e., patients who exclusively complied with the treatment. Missing data will be handled with pair-wise exclusion.

The primary analysis will use Student's t-test for the 2 independent groups, surgical or non-surgical treatment, to compare group difference in ODI after 1 year. Subgroup analyses will be used for covariates age, sex, fracture type (A3 vs A4), presence of nerve root symptoms, degree of fracture comminution (47), degree of vertebral compression, kyphosis, and integrity of the PLC (intact vs. indeterminate). When the trial has finished there might be a situation where we find a small but statistically significant difference between groups in ODI that is less than the estimated MCID of 10. However, statistical significance does not equal clinical relevance. If we find be dichotomized in a similar matter based on the results of ongoing studies. Treatment and the same covariates as the primary analysis will be used as independent variables to identify which predictors are important for the outcome. Depending on distribution, Spearman's or Pearson's correlation will be used to test the bivariate correlation between continuous variables, i.e., comparing PROMs with each other.

An independent statistician will perform the statistical analysis. All statistical analyses will be performed without knowledge of the actual treatment (surgical or non-surgical) to minimize bias (56).

The ICER of surgical and non-surgical care will be estimated by combining cost and QALY (from EQ-5D-5L). To quantify the uncertainty around estimates, bootstrapping will be used. The ICER will be compared with commonly used estimates of ICERs of other interventions (mainly surgeries and pharmaceutical interventions). Given the uncertainty around the official thresholds of willingness to pay for healthcare interventions, a cost-effectiveness acceptability curve will be drawn, showing the likelihood of the surgical intervention being costeffective given different levels of willingness to pay.

\section{Ethics, data sharing plan, funding, registration, and potential conflicts of interests}

The study has been approved by the Swedish Ethical Review Authority (Approval No: 2021-00011). The study will be performed in accordance with the published protocol, the latest version of the Declaration of Helsinki, and applicable regulatory requirements. Should any substantial protocol amendment occur, the Ethics Committee concerned will be informed and asked for its opinion, prior to implementation of the amended protocol, as to whether a full re-evaluation of the ethical aspects of the study is necessary by the committee. Any changes to the protocol will be presented in publications of study findings. 
At the conclusion of the study, the occurrence of any protocol deviations will be determined. After these actions have been completed and the database has been declared to be complete and accurate, it will be locked and available for data analysis. Datasets derived from the current study that are needed to replicate the findings will be made publicly available.

The trial is funded by a grant from the Swedish Research Council (Grant No: 2020-00493). The funding agency has no role in study design, data collection, interpretation of data, or writing of manuscripts.

The trial is registered at clinicaltrials.gov, NCT05003180 (August 12, 2021).

The author PF has received occasional lecture fees from Johnson \& Johnson and in the SunBurst study group LB has received a research grant (unrelated to the current project) and lecture fees from Medtronic. All other authors have no conflict of interest.

\section{Study timetable}

The study started on September 1, 2021. The study is estimated to continue for 4 years but may be delayed in order to recruit the necessary number of patients. If delays occur, the SunBurst study group plans to reach out to additional centers internationally for the possibility of study expansion. Last follow-up for the primary outcome will be 1 year after last subject recruitment. The results of the study will be submitted for peer-reviewed publication and presented at international conferences. Further registry outcome data is planned to be collected 5 and 10 years after last subject recruitment. The study is estimated to have come to an end in December 2036.

\section{Discussion}

SunBurst will be the 1st nationwide R-RCT on vertebral fractures through the SFR platform with the aim of answering the question: Is surgical or non-surgical treatment the most appropriate for patients with thoracolumbar burst fractures without neurological deficit and without complete rupture of the PLC? It will be the largest RCT to date comparing surgical with non-surgical care in patients with thoracolumbar burst fractures.

Although being the gold standard for comparing treatments in medicine, RCTs come at great cost and a heavy administrative workload. A register-based RCT has the advantage of greatly lowering cost by using an established register and simplifies recruitment of patients using a webbased platform. An R-RCT can facilitate and accelerate recruitment of study participants, making it possible to carry out large, adequately powered studies in a relatively short time. Another advantage with the R-RCTs with a pragmatic approach is that it is more likely to include a more unselected group of patients compared with ordinary RCTs, which more correctly reflects the real population and potentially improves external validity (27). Although this study uses the infrastructure of the SFR, other data including PROMs is collected outside the register; we may therefore also call this study a hybrid R-RCT.

Like previous studies on thoracolumbar burst fractures comparing a non-surgical with a surgical alternative, blinding the subjects is not possible. This may potentially introduce several confounders. However, any baseline confounder is likely to be evenly distributed between the study groups. The PROMs will be assessed by the patients themselves, without the help of healthcare personnel or the researchers involved. The placebo effect in surgery is a potential confounding factor. It has been shown to have importance in short-term efficacy, especially concerning patients with chronic pain (57). Hopefully, this effect will be minimized because the patients have an acute condition and follow-ups up to 1 year after the accident, after which a placebo effect may be attenuated.

A limitation of our study is that the clinically relevant group difference for ODI is not established, which makes the power calculation slightly insecure. Our sample size calculation is based on the individual MCID $(\mathbf{5 5 , 5 8 )}$. We aim to include 202 patients, which we hope will be more than enough to discover any relevant differences between treatments.

Measuring sick leave and return to work may prove to be an important factor in determining which treatment is the most cost-effective on a societal level. Our study participants, aged 18 to 66 at the time of injury, will be within working age in Sweden. Nevertheless, it cannot be excluded that the given treatment will affect the recommendations on sick leave, for example reasoning that surgery is a more invasive and extensive treatment resulting in prolonged sick leave. This may bias the evaluations on cost. To increase the knowledge on sick leave patterns after vertebral fractures a pilot observational study based on registry data is planned.

In summary, we hope that this R-RCT on thoracolumbar burst fractures will be able to provide important evidence as to which treatment leads to better patient-reported outcome as well as better knowledge of the health economic impact each treatment has for both the patient and society.

The authors would like to thank Linda Magné and Marko Poikkimäki for the technical solutions behind the study platform in the SFR.

PF, PG, SM, and OW designed the trial and shared in reviewing the manuscript. Ethical applications were handled by PG. SB drafted the manuscript. All authors and the SunBurst study group have contributed to the contents of the protocol during meetings and mail conversations. All authors and the SunBurst study group have given their final approval of the version to be published and agree to be accountable for all aspects of the work. The final report will be written by the authors of this article.

1. Denis $\mathbf{F}$. The three column spine and its significance in the classification of acute thoracolumbar spinal injuries. Spine (Phila Pa 1976) 1983; 8(8): 817-31. 
2. Rajasekaran S, Kanna RM, Shetty A P. Management of thoracolumbar spine trauma: an overview. Indian J Orthop 2015; 49(1): 72-82.

3. Schouten R, Lewkonia P, Noonan V K, Dvorak M F, Fisher C G. Expectations of recovery and functional outcomes following thoracolumbar trauma: an evidence-based medicine process to determine what surgeons should be telling their patients. J Neurosurg Spine 2015; 22(1): 101-11.

4. Andress H J, Braun H, Helmberger T, Schürmann M, Hertlein H, Hartl W H. Long-term results after posterior fixation of thoraco-lumbar burst fractures. Injury 2002; 33(4): 357-65.

5. McLain R F. Functional outcomes after surgery for spinal fractures: return to work and activity. Spine (Phila Pa 1976) 2004; 29(4): 470-7; discussion Z6.

6. Briem D, Behechtnejad A, Ouchmaev A, Morfeld M, SchermellehEngel K, Amling M, et al. Pain regulation and health-related quality of life after thoracolumbar fractures of the spine. Eur Spine J 2007; 16(11): 1925-33.

7. Moller A, Hasserius R, Redlund-Johnell I, Ohlin A, Karlsson M K. Nonoperatively treated burst fractures of the thoracic and lumbar spine in adults: a 23- to 41-year follow-up. Spine J 2007; 7(6): 701-7.

8. Wood K B, Buttermann G R, Phukan R, Harrod C C, Mehbod A, Shannon B, et al. Operative compared with nonoperative treatment of a thoracolumbar burst fracture without neurological deficit: a prospective randomized study with follow-up at sixteen to twenty-two years. J Bone Joint Surg Am 2015; 97(1): 3-9.

9. Marek A P, Morancy J D, Chipman J G, Nygaard R M, Roach R M, Loor M M. Long-term functional outcomes after traumatic thoracic and lumbar spine fractures. Am Surg 2018; 84(1): 20-7.

10. Gnanenthiran $\mathbf{S} \mathbf{R}$, Adie $\mathbf{S}$, Harris I A. Nonoperative versus operative treatment for thoracolumbar burst fractures without neurologic deficit: a meta-analysis. Clin Orthop Relat Res 2012; 470(2): 567-77.

11. Wood K B, Li W, Lebl D R, Ploumis A. Management of thoracolumbar spine fractures. Spine J 2014; 14(1): 145-64.

12. Vaccaro A R, Schroeder G D, Kepler C K, Cumhur Oner F, Vialle L R, Kandziora F, et al. The surgical algorithm for the AOSpine thoracolumbar spine injury classification system. Eur Spine J 2016; 25(4): 1087-94.

13. Oner C, Rajasekaran S, Chapman J R, Fehlings M G, Vaccaro A R, Schroeder G D, et al. Spine trauma-what are the current controversies? J Orthop Trauma 2017; 31(Suppl. 4): S1-S6.

14. Yi L, Jingping B, Gele J, Baoleri X, Taixiang W. Operative versus nonoperative treatment for thoracolumbar burst fractures without neurological deficit. Cochrane Database Syst Rev 2006(4): Cd005079.

15. Dai L Y, Jiang S D, Wang X Y, Jiang L S. A review of the management of thoracolumbar burst fractures. Surg Neurol 2007; 67(3): 221-31; discussion 31 .

16. van der Roer N, de Lange E S, Bakker F C, de Vet H C, van Tulder M W. Management of traumatic thoracolumbar fractures: a systematic review of the literature. Eur Spine J 2005; 14(6): 527-34.

17. Wood K, Buttermann G, Mehbod A, Garvey T, Jhanjee R, Sechriest V. Operative compared with nonoperative treatment of a thoracolumbar burst fracture without neurological deficit: a prospective, randomized study. J Bone Joint Surg Am 2003; 85(5): 773-81.

18. Siebenga J, Leferink V J, Segers M J, Elzinga M J, Bakker F C, Haarman $\mathbf{H} \mathbf{J}$, et al. Treatment of traumatic thoracolumbar spine fractures: a multicenter prospective randomized study of operative versus nonsurgical treatment. Spine (Phila Pa 1976) 2006; 31(25): 2881-90.

19. Abudou M, Chen X, Kong X, Wu T. Surgical versus non-surgical treatment for thoracolumbar burst fractures without neurological deficit. Cochrane Database Syst Rev 2013(6): Cd005079.

20. Ghobrial G M, Maulucci C M, Maltenfort M, Dalyai R T, Vaccaro A R, Fehlings M G, et al. Operative and nonoperative adverse events in the management of traumatic fractures of the thoracolumbar spine: a systematic review. Neurosurg Focus 2014; 37(1): E8.
21. Rometsch E, Spruit M, Härtl R, McGuire RA, Gallo-Kopf B S, Kalampoki V, et al. Does operative or nonoperative treatment achieve better results in A3 and A4 spinal fractures without neurological deficit?: systematic literature review with meta-analysis. Global Spine J 2017; 7(4): $350-72$

22. Wallace N, McHugh M, Patel R, Aleem I S. Effects of bracing on clinical and radiographic outcomes following thoracolumbar burst fractures in neurologically intact patients: a meta-analysis of randomized controlled trials. JBJS Rev 2019; 7(9): e9.

23. Emilsson L, Lindahl B, Köster M, Lambe M, Ludvigsson J F. Review of 103 Swedish healthcare quality registries. J Intern Med 2015; 277(1): 94-136.

24. Wennergren D, Möller M. Implementation of the Swedish Fracture Register. Unfallchirurg 2018; 121(12): 949-55.

25. Wolf $\mathbf{O}$, Sjöholm P, Hailer N P, Möller M, Mukka S. Study protocol: HipSTHeR - a register-based randomised controlled trial-hip screws or (total) hip replacement for undisplaced femoral neck fractures in older patients. BMC Geriatr 2020; 20(1): 19.

26. Wolf O, Mukka S, Notini M, Möller M, Hailer N P. Study protocol: the DUALITY trial - a register-based, randomized controlled trial to investigate dual mobility cups in hip fracture patients. Acta Orthop 2020; 91(5): 506-13.

27. James S, Rao S V, Granger C B. Registry-based randomized clinical trials:a new clinical trial paradigm. Nat Rev Cardiol 2015; 12(5): 312-6.

28. Chan A W, Tetzlaff J M, Altman D G, Laupacis A, Gøtzsche P C, Krleža-Jerić K, et al. SPIRIT 2013 statement: defining standard protocol items for clinical trials. Ann Intern Med 2013; 158(3): 200-7.

29. Vaccaro A R, Oner C, Kepler C K, Dvorak M, Schnake K, Bellabarba C, et al. AOSpine thoracolumbar spine injury classification system: fracture description, neurological status, and key modifiers. Spine (Phila Pa 1976) 2013 ; 38(23): 2028-37.

30. Reinhold M, Audigé L, Schnake K J, Bellabarba C, Dai L Y, Oner F C. AO spine injury classification system: a revision proposal for the thoracic and lumbar spine. Eur Spine J 2013; 22(10): 2184-201.

31. Vaccaro A R, Rihn J A, Saravanja D, Anderson D G, Hilibrand A S, Albert T J, et al. Injury of the posterior ligamentous complex of the thoracolumbar spine: a prospective evaluation of the diagnostic accuracy of magnetic resonance imaging. Spine (Phila Pa 1976) 2009; 34(23): E841-7.

32. Ni W F, Huang Y X, Chi Y L, Xu H Z, Lin Y, Wang X Y, et al. Percutaneous pedicle screw fixation for neurologic intact thoracolumbar burst fractures. J Spinal Disord Tech 2010; 23(8): 530-7.

33. Chi J H, Eichholz K M, Anderson P A, Arnold P M, Dailey A T, Dhall S S, et al. Congress of Neurological Surgeons systematic review and evidence-based guidelines on the evaluation and treatment of patients with thoracolumbar spine trauma: novel surgical strategies. Neurosurgery 2019; 84(1): E59-e62.

34. Rajasekaran S. Thoracolumbar burst fractures without neurological deficit: the role for conservative treatment. Eur Spine J 2010; 19(Suppl. 1): S40-7.

35. Fairbank J C, Couper J, Davies J B, O'Brien J P. The Oswestry low back pain disability questionnaire. Physiotherapy 1980; 66(8): 271-3.

36. Fairbank J C, Pynsent P B. The Oswestry Disability Index. Spine (Phila Pa 1976) 2000; 25(22): 2940-52; discussion 52.

37. Roland M, Fairbank J. The Roland-Morris Disability Questionnaire and the Oswestry Disability Questionnaire. Spine (Phila Pa 1976) 2000; 25(24): 3115-24.

38. Strömqvist B, Fritzell P, Hägg $\mathbf{O}$, Jönsson B. The Swedish Spine Register: development, design and utility. Eur Spine J 2009; 18(Suppl. 3): 294-304.

39. Fairbank J C. Use and abuse of Oswestry Disability Index. Spine (Phila Pa 1976) 2007; 32(25): 2787-9.

40. Vianin M. Psychometric properties and clinical usefulness of the Oswestry Disability Index. J Chiropr Med 2008; 7(4): 161-3.

41. Endler P, Ekman P, Ljungqvist H, Brismar T B, Gerdhem P, Möller H. Long-term outcome after spinal fusion for isthmic spondylolisthesis in adults. Spine J 2019; 19(3): 501-8. 
42. Tonosu J, Takeshita K, Hara N, Matsudaira K, Kato S, Masuda K, et al. The normative score and the cut-off value of the Oswestry Disability Index (ODI). Eur Spine J 2012; 21(8): 1596-602.

43. Wei F X, Liu S Y, Liang C X, Li H M, Long H Q, Yu B S, et al. Transpedicular fixation in management of thoracolumbar burst fractures: monosegmental fixation versus short-segment instrumentation. Spine (Phila Pa 1976) 2010; 35(15): E714-20

44. Ponzer S, Skoog A, Bergström G. The Short Musculoskeletal Function Assessment Questionnaire (SMFA): cross-cultural adaptation, validity, reliability and responsiveness of the Swedish SMFA (SMFA-Swe). Acta Orthop Scand 2003; 74(6): 756-63.

45. Herdman M, Gudex C, Lloyd A, Janssen M, Kind P, Parkin D, et al. Development and preliminary testing of the new five-level version of EQ-5D (EQ-5D-5L). Qual Life Res 2011; 20(10): 1727-36.

46. Burström K, Teni F S, Gerdtham U G, Leidl R, Helgesson G, Rolfson O, et al. Experience-based Swedish TTO and VAS value sets for EQ5D-5L health states. Pharmacoeconomics 2020; 38(8): 839-56.

47. McCormack T, Karaikovic E, Gaines R W. The load sharing classification of spine fractures. Spine (Phila Pa 1976) 1994; 19(15): 1741-4.

48. National Board of Health and Welfare. The National Patient Register 2019 [updated 2019/05/20. Available from: https://www.socialstyrelsen. se/en/statistics-and-data/registers/register-information/the-nationalpatient-register/.

49. National Board of Health and Welfare. The Swedish Prescribed Drug Register 2020 [updated 2020/08/21]. Available from: https://www.socialstyrelsen.se/en/statistics-and-data/registers/register-information/theswedish-prescribed-drug-register/.

50. National Board of Health and Welfare. Dödsorsaksregistret 2018. Available from: https://www.socialstyrelsen.se/statistik-och-data/register/alla-register/dodsorsaksregistret/.
51. Rampersaud Y R, Moro E R, Neary M A, White K, Lewis S J, Massicotte $\mathbf{E} \mathbf{M}$, et al. Intraoperative adverse events and related postoperative complications in spine surgery: implications for enhancing patient safety founded on evidence-based protocols. Spine (Phila Pa 1976) 2006; 31(13): 1503-10.

52. Landriel Ibañez F A, Hem S, Ajler P, Vecchi E, Ciraolo C, Baccanelli $\mathbf{M}$, et al. A new classification of complications in neurosurgery. World Neurosurg 2011; 75(5-6): 709-15; discussion 604-11.

53. Swedish Social Insurance Agency. Försäkringskassan 2021. Available from: https://www.forsakringskassan.se/english.

54. Statistics Sweden. Longitudinal integrated database for health insurance and labour market studies (LISA) 2021. Available from: https://www.scb.se/en/services/ordering-data-and-statistics/orderingmicrodata/vilka-mikrodata-finns/longitudinella-register/longitudinalintegrated-database-for-health-insurance-and-labour-market-studieslisa/.

55. Copay A G, Glassman S D, Subach B R, Berven S, Schuler T C, Carreon L Y. Minimum clinically important difference in lumbar spine surgery patients: a choice of methods using the Oswestry Disability Index, Medical Outcomes Study questionnaire Short Form 36, and pain scales. Spine J 2008; 8(6): 968-74.

56. MacCoun R, Perlmutter S. Blind analysis: Hide results to seek the truth. Nature 2015; 526(7572): 187-9.

57. Jonas W B, Crawford C, Colloca L, Kaptchuk T J, Moseley B, Miller F G, et al. To what extent are surgery and invasive procedures effective beyond a placebo response? A systematic review with meta-analysis of randomised, sham controlled trials. BMJ Open 2015; 5(12): e009655.

58. Hägg $\mathbf{O}$, Fritzell $\mathbf{P}$, Nordwall $\mathbf{A}$. The clinical importance of changes in outcome scores after treatment for chronic low back pain. Eur Spine $\mathbf{J}$ 2003; 12(1): 12-20.

\section{Supplementary data}

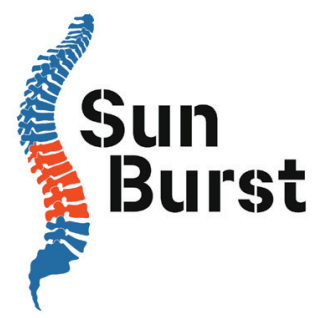

Study logo. Design by Magnus Widlund.

\section{Appendix 1: ICD codes}

\begin{tabular}{ll}
\hline \multicolumn{2}{l}{ Diagnostic ICD-10 codes $\quad$ Descriptions in English } \\
\hline $\begin{array}{ll}\text { Medical complications } \\
\text { I26 }\end{array}$ & I26.0: Pulmonary embolism with acute cor pulmonale. I26.9: Pulmonary embolism without acute cor pulmonale \\
I80 & Phlebitis and thrombophlebitis \\
I20-I25 & Ischemic heart diseases including detailed descriptions \\
N39.0 & Urinary tract infection, site not specified \\
N30.9 & Cystitis, unspecified \\
N30.0 & Acute cystitis \\
J13-J17.0+J18 & Influenza and pneumonia (unspecified organism and certain bacterial pneumonias) \\
Covariates & Ischemic heart diseases including detailed descriptions \\
I20-I25 & Diabetes mellitus \\
E10-E14 & Complications of procedures, not elsewhere classified \\
Surgical complications \\
T81 & Nonunion of fracture (pseudarthrosis) \\
M84.1 & Mechanical complication of internal fixation device of other bones \\
T84.2 & Other complications of surgical and medical care, not elsewhere classified, including detailed descriptions \\
T88 &
\end{tabular}

